\title{
GRAVITATIONAL FARADAY EFFECT PRODUCED BY A RING LASER
}

DAVID ERIC COX, JAMES G. O'BRIEN, RONALD L. MALLETT,

AND CHANDRA ROYCHOUDHURI

Using the linearized Einstein gravitational field equations and the Maxwell field equations it is shown that the plane of polarization of an electromagnetic wave is rotated by the gravitational field created by the electromagnetic radiation of a ring laser. It is further shown that this gravitational Faraday effect shares many of the properties of the standard electromagnetic Faraday effect. An experimental arrangement is then suggested for the observation of this gravitational Faraday effect induced by the ring laser.

Key Words: general relativity; gravitation; lasers; Maxwell's equations; Faraday effect. 


\section{INTRODUCTION}

Frame dragging of a satellite in polar orbit induced by the gravitational field of a massive rotating body ${ }^{(1)}$ was one of the earliest post Newtonian predictions of Einstein's general theory of relativity. More recently one of us ${ }^{(2)}$ showed that the gravitational field generated by the electromagnetic radiation in a ring laser results in the frame dragging precession of a spinning neutral massive particle. The gravitational field generated by either a massive rotating body or a circulating light beam has many properties similar to those of a magnetic field. One of the most interesting phenomena of classical electrodynamics is the Faraday effect. In this effect, the plane of polarization of an electromagnetic wave is rotated while traversing a dielectric in the presence of a magnetic field along the direction of the field. This suggests that there may be a gravitational Faraday effect associated with the gravitational field of a rotating massive body. This was in fact shown to be the case by Balazs ${ }^{(3)}$.

In this paper, the Maxwell field equations are solved for an electromagnetic wave in the presence of the gravitational field of a ring laser. It is shown that a gravitational Faraday effect is induced, resulting in the rotation of the plane of polarization of an electromagnetic wave. This gravitational Faraday effect is shown to have similarities to the electromagnetic Faraday effect. A possible experiment is discussed for the observation of the gravitational Faraday effect produced by the ring laser. 


\section{MAXWELL EQUATIONS IN WEAK GRAVIATIONAL FIELD}

The weak-field linear approximation of the Einstein gravitational field equations is given by

$$
\partial_{\alpha} \partial^{\alpha} h^{\mu \nu}=-k T^{\mu \nu}
$$

with the energy-momentum tensor for an electromagnetic field,

$$
T^{\mu \nu}=-\frac{1}{4 \pi}\left(F^{\mu \alpha} F_{\alpha}^{\nu}-\frac{1}{4} \eta^{\mu \nu} F^{\alpha \beta} F_{\alpha \beta}\right)
$$

where $h_{\mu \nu}$ is the weak gravitational field. Using the notation given in Landau and Lifshitz $^{(4)}$ for the covariant Maxwell equations, one finds that the modified fields in an external gravitational field are given by

$$
\begin{aligned}
& B^{i j}=\frac{H^{i j}}{\sqrt{h}}+E^{i} g^{j}-E^{j} g^{i} \text { and } \\
& D_{i}=\frac{E_{i}}{\sqrt{h}}+g^{k} H_{i k},
\end{aligned}
$$

or, in vector notation,

$$
\begin{aligned}
& \boldsymbol{D}=\frac{\boldsymbol{E}}{\sqrt{h}}+\boldsymbol{H} \times \boldsymbol{g} . \\
& \boldsymbol{B}=\frac{\boldsymbol{H}}{\sqrt{h}}+\boldsymbol{g} \times \boldsymbol{E} .
\end{aligned}
$$

where

$$
\begin{aligned}
& h=\sqrt{g_{00}} . \\
& g_{i} \equiv-\frac{g_{0 i}}{g_{00}} . \\
& \gamma_{i j} \equiv h g_{i} g_{j}-g_{i j} .
\end{aligned}
$$


This leads to the four Maxwell equations in a weak gravitational field given by

$$
\begin{aligned}
& \boldsymbol{\nabla} \cdot \boldsymbol{D}=4 \pi \rho . \\
& \boldsymbol{\nabla} \cdot \boldsymbol{B}=0 . \\
& \frac{1}{c \sqrt{\gamma}} \frac{\partial \sqrt{\gamma} \boldsymbol{B}}{\partial t}+\boldsymbol{\nabla} \times \boldsymbol{E}=\mathbf{0} . \\
& \boldsymbol{\nabla} \times \boldsymbol{H}-\frac{1}{c \sqrt{\gamma}} \frac{\partial \sqrt{\gamma} \boldsymbol{D}}{\partial t}=\frac{4 \pi}{c} \boldsymbol{j} .
\end{aligned}
$$

\section{GRAVITATIONAL FARADAY EFFECT AND THE PLANE OF POLARIZATION OF A LIGHT RAY}

Consider a ring laser which lies in the $x-y$ plane, with one corner at the origin as pictured in Figure 1. The ring laser has linear dimension $a$ and a linear energy density of $\lambda$. We consider a plane polarized wave very far from the laser ring, aligned along on the vertical through the center of the ring laser, and pointing in the positive $z$ direction. Our goal is to determine the polarization angle of the incident beam far from the laser ring (see Figure 1).

The metric produced by the ring laser configuration was determined ${ }^{(2)}$ to be

$$
\begin{aligned}
& \Phi_{(1)} \equiv \ln \frac{a-x+\sqrt{(x-a)^{2}+y^{2}+z^{2}}}{\sqrt{x^{2}+y^{2}+z^{2}}-x} . \\
& \Phi_{(2)} \equiv \ln \frac{a-y+\sqrt{(x-a)^{2}+(y-a)^{2}+z^{2}}}{\sqrt{(x-a)^{2}+y^{2}+z^{2}}-y} .
\end{aligned}
$$




$$
\begin{aligned}
& \Phi_{(3)} \equiv \ln \frac{a-x+\sqrt{(x-a)^{2}+(y-a)^{2}+z^{2}}}{\sqrt{x^{2}+(y-a)^{2}+z^{2}}-x} . \\
& \Phi_{(4)} \equiv \ln \frac{a-y+\sqrt{x^{2}+(y-a)^{2}+z^{2}}}{\sqrt{x^{2}+y^{2}+z^{2}}-y} . \\
& h^{\mu \nu}=-\frac{k \lambda}{4 \pi}\left(\begin{array}{cccc}
\Phi_{(1)}+\Phi_{(2)}+\Phi_{(3)}+\Phi_{(4)} & \Phi_{(1)}-\Phi_{(3)} & \Phi_{(2)}-\Phi_{(4)} & 0 \\
\Phi_{(1)}-\Phi_{(3)} & \Phi_{(1)}+\Phi_{(3)} & 0 & 0 \\
\Phi_{(2)}-\Phi_{(4)} & 0 & \Phi_{(2)}+\Phi_{(4)} & 0 \\
0 & 0 & 0 & 0
\end{array}\right) \\
& =-\frac{2 G \lambda}{c^{4}}\left(\begin{array}{cccc}
\Phi_{(1)}+\Phi_{(2)}+\Phi_{(3)}+\Phi_{(4)} & \Phi_{(1)}-\Phi_{(3)} & \Phi_{(2)}-\Phi_{(4)} & 0 \\
\Phi_{(1)}-\Phi_{(3)} & \Phi_{(1)}+\Phi_{(3)} & 0 & 0 \\
\Phi_{(2)}-\Phi_{(4)} & 0 & \Phi_{(2)}+\Phi_{(4)} & 0 \\
0 & 0 & 0 & 0
\end{array}\right) . \\
& h_{\mu \nu}=\eta_{\mu \alpha} \eta_{\nu \beta} h^{\alpha \beta}=h^{\mu \nu} . \\
& g_{\mu \nu}=\eta_{\mu \nu}+h_{\mu \nu} .
\end{aligned}
$$

This gives

$$
\begin{aligned}
& h=1-\frac{2 G \lambda}{c^{4}}\left[\Phi_{(1)}+\Phi_{(2)}+\Phi_{(3)}+\Phi_{(4)}\right] . \\
& \boldsymbol{g}=-\frac{2 G \lambda}{c^{4}}\left\{\left[\Phi_{(1)}-\Phi_{(3)}\right] \boldsymbol{e}_{\hat{x}}-\left[\Phi_{(2)}-\Phi_{(4)}\right] \boldsymbol{e}_{\hat{y}}\right\} . \\
& \gamma=\left\{1+\frac{2 G \lambda}{c^{4}}\left[\Phi_{(1)}+\Phi_{(3)}\right]\right\}\left\{1+\frac{2 G \lambda}{c^{4}}\left[\Phi_{(2)}+\Phi_{(4)}\right]\right\} .
\end{aligned}
$$

Since this is a linear approximation, we are assuming that

$$
\frac{2 G \lambda}{c^{4}} \sum_{\substack{i \\ \text { Any combination } i}} \Phi_{(i)} \ll 1 \text {. }
$$


This leads to the necessary approximations:

$$
\begin{aligned}
& h=1 \\
& \boldsymbol{g}=g_{x} \boldsymbol{e}_{x}+g_{y} \boldsymbol{e}_{y} ; \quad\left|g_{x}\right|,\left|g_{y}\right| \ll 1 \\
& \gamma=1 .
\end{aligned}
$$

Using these approximations in Equations (10) through (13), for light moving in a vacuum, gives

$$
\begin{aligned}
& \boldsymbol{D}=\boldsymbol{E}+\boldsymbol{H} \times \boldsymbol{g} . \\
& \boldsymbol{B}=\boldsymbol{H}+\boldsymbol{g} \times \boldsymbol{E} . \\
& \frac{1}{c} \frac{\partial \boldsymbol{B}}{\partial t}+\boldsymbol{\nabla} \times \boldsymbol{E}=\mathbf{0} . \\
& \boldsymbol{\nabla} \cdot \boldsymbol{B}=0 . \\
& \boldsymbol{\nabla} \cdot \boldsymbol{D}=0 . \\
& \boldsymbol{\nabla} \times \boldsymbol{H}-\frac{1}{c} \frac{\partial \boldsymbol{D}}{\partial t}=\mathbf{0} .
\end{aligned}
$$

Decoupling these field equations, making use of the fact that $\boldsymbol{\nabla} \cdot \boldsymbol{g}=0$, and neglecting higher orders of $g \equiv|\boldsymbol{g}|$ leads to

$$
\begin{aligned}
& \frac{1}{c} \frac{\partial \boldsymbol{B}}{\partial t}+\nabla \times \boldsymbol{D}-\boldsymbol{B}(\boldsymbol{\nabla} \cdot \boldsymbol{g})-(\boldsymbol{g} \cdot \boldsymbol{\nabla}) \boldsymbol{B}+(\boldsymbol{B} \cdot \boldsymbol{\nabla}) \boldsymbol{g}=\mathbf{0} . \\
& \boldsymbol{\nabla} \times \boldsymbol{B}-(\boldsymbol{D} \cdot \boldsymbol{\nabla}) \boldsymbol{g}+(\boldsymbol{g} \cdot \boldsymbol{\nabla}) \boldsymbol{D}+\boldsymbol{B}(\boldsymbol{\nabla} \cdot \boldsymbol{g})-\frac{1}{c} \frac{\partial \boldsymbol{D}}{\partial t}=\mathbf{0} .
\end{aligned}
$$


In order to solve Equations (31) and (32), we take the following into consideration: First, The incident beam is plane polarized:

$$
\begin{aligned}
& \boldsymbol{D}=D_{x} \boldsymbol{e}_{\hat{x}}+D_{y} \boldsymbol{e}_{\hat{y}} . \\
& \boldsymbol{B}=B_{x} \boldsymbol{e}_{\hat{x}}+B_{y} \boldsymbol{e}_{\hat{y}} .
\end{aligned}
$$

Next, for any arbitrary vector, $\boldsymbol{t}$, expressions of the form $(\boldsymbol{t} \cdot \boldsymbol{\nabla}) \boldsymbol{g}$ can be simplified when examining effects far from the ring laser since

$$
(\boldsymbol{t} \cdot \boldsymbol{\nabla}) \boldsymbol{g}=\left(t_{x} \frac{\partial g_{x}}{\partial x}+t_{y} \frac{\partial g_{x}}{\partial y}\right) \boldsymbol{e}_{\hat{x}}+\left(t_{x} \frac{\partial g_{y}}{\partial x}+t_{y} \frac{\partial g_{y}}{\partial y}\right) \boldsymbol{e}_{\hat{y}} .
$$

Neglecting terms with of order $r^{-4}$ or smaller:

$$
\begin{array}{ll}
\frac{\partial g_{x}}{\partial x} \underset{r \rightarrow \infty}{\longrightarrow} 0 . & \frac{\partial g_{x}}{\partial y} \underset{r \rightarrow \infty}{\longrightarrow} \frac{2 G \lambda x y}{c^{4} r^{3}} . \\
\frac{\partial g_{y}}{\partial x} \underset{r \rightarrow \infty}{\longrightarrow}-\frac{2 G \lambda x y}{c^{4} r^{3}} . & \frac{\partial g_{y}}{\partial y} \underset{r \rightarrow \infty}{\longrightarrow} 0 .
\end{array}
$$

Therefore, on the vertical through the center of the ring laser,

$$
\begin{gathered}
\sigma(r) \equiv-\frac{G \lambda a^{2}}{2 c^{4} r^{3}} . \\
(\boldsymbol{t} \cdot \nabla) \boldsymbol{g} \underset{r \rightarrow \infty}{\longrightarrow}-t_{y} \sigma(r) \boldsymbol{e}_{\hat{x}}+t_{x} \sigma(r) \boldsymbol{e}_{\hat{y}} .
\end{gathered}
$$

By approximating the ray of light as a plane wave,

$$
\begin{aligned}
& \frac{\partial \boldsymbol{D}}{\partial x}=\frac{\partial \boldsymbol{D}}{\partial y}=0 . \\
& \frac{\partial \boldsymbol{B}}{\partial x}=\frac{\partial \boldsymbol{B}}{\partial y}=0 .
\end{aligned}
$$


Applying these three considerations to Equations (31) and (32) gives

$$
\begin{aligned}
& \frac{1}{c} \frac{\partial B_{x}}{\partial t}-\frac{\partial D_{y}}{\partial z}-B_{y} \sigma(r)=0 . \\
& \frac{1}{c} \frac{\partial B_{y}}{\partial t}+\frac{\partial D_{x}}{\partial z}+B_{x} \sigma(r)=0 . \\
& -\frac{\partial B_{y}}{\partial z}+D_{y} \sigma(r)-\frac{1}{c} \frac{\partial D_{x}}{\partial t}=0 . \\
& \frac{\partial B_{x}}{\partial z}-D_{x} \sigma(r)-\frac{1}{c} \frac{\partial D_{y}}{\partial t}=0 .
\end{aligned}
$$

We can remove the time dependence and convert these to total differential equations by making the substitutions

$$
\begin{aligned}
& D_{ \pm}(z) e^{-i \omega t} \equiv D_{x} \pm i D_{y} . \\
& B_{ \pm}(z) e^{-i \omega t} \equiv B_{x} \pm i B_{y} .
\end{aligned}
$$

This yields

$$
\begin{aligned}
& \frac{d D_{ \pm}}{d z}+\left[\sigma(r) \mp \frac{\omega}{c}\right] B_{ \pm}=0 . \\
& \frac{d B_{ \pm}}{d z}-\left[\sigma(r) \mp \frac{\omega}{c}\right] D_{ \pm}=0 .
\end{aligned}
$$

We now assume that the gravitational field from the ring laser will only slightly modify the plane wave and that this modification varies very slowly as one moves away or towards the ring laser, that is to say a pair of functions, $\ell_{ \pm}(z)$, exist such that

$$
\begin{aligned}
D_{ \pm} & =d_{ \pm} e^{i\left[\ell_{ \pm}(z)+\frac{\omega}{c} z\right]} . \\
B_{ \pm} & =b_{ \pm} e^{i\left[\ell_{ \pm}(z)+\frac{\omega}{c} z\right]} \\
\frac{d \ell_{ \pm}}{d z} & \ll 1 .
\end{aligned}
$$


Substituting these into Equations (46) and (47) while neglecting higher-order terms of $d \ell / d z$ and $\sigma$ gives

$$
\frac{d \ell_{ \pm}}{d z} \pm \sigma(r)=0
$$

Integrating this equation yields the result for the modification of the fields as

$$
\ell_{ \pm}=\mp \int_{-\infty}^{z} \sigma\left(r^{\prime}\right) d z^{\prime}=\frac{G \lambda}{c^{4}}\left[1+\frac{\operatorname{sgn} z}{\sqrt{1+\frac{1}{2}\left(\frac{a}{z}\right)^{2}}}\right]
$$

We now consider the physical implications. Taking the real part of $\boldsymbol{D}$,

$$
\begin{aligned}
D_{x} & =d_{0} \cos \left(\frac{\omega}{c} z-\omega t\right) \cos \ell_{+} . \\
D_{y} & =d_{0} \cos \left(\frac{\omega}{c} z-\omega t\right) \sin \ell_{+} .
\end{aligned}
$$

Hence, the angle of polarization will be

$$
\begin{aligned}
\varphi & =\tan ^{-1} \frac{D_{y}}{D_{x}} \\
& =\tan ^{-1} \tan \ell_{+} \\
& =\ell_{+} .
\end{aligned}
$$

Thus, we see that the change in the angle of polarization is the previously calculated $\ell$. Letting the $z$ direction increase without bound, we see that the polarization shift of an incident beam due to the ring laser is the remarkably simple result:

$$
\Delta \varphi=\frac{2 G \lambda}{c^{4}} .
$$

This demonstrates the gravitational analog of the Faraday effect produced by a ring laser on an incident, plane polarized light beam. There exists a particularly important consequence, which closely resembles that of the classical Faraday effect. Let us now examine the effect of placing the ray infinitely above the ring laser, 
heading down, the $\omega z / c$ term in the exponentials in Equations (48) and (49) become $-\omega z / c$ and the lower integration limit in Equation (51) changes from $-\infty$ to $\infty$ :

$$
\begin{aligned}
& D_{ \pm}=d_{ \pm} e^{i\left[\ell_{ \pm}(z)-\frac{\omega}{c} z\right]} . \\
& B_{ \pm}=b_{ \pm} e^{i\left[\ell_{ \pm}(z)-\frac{\omega}{c} z\right]} . \\
& \frac{d \ell_{ \pm}}{d z} \mp \sigma(r)=0 .
\end{aligned}
$$

Thus, integrating as before, from the new limits, we find

$$
\begin{aligned}
\ell_{ \pm} & = \pm \int_{\infty}^{z} \sigma\left(r^{\prime}\right) d z^{\prime}=\mp \frac{G \lambda}{c^{4}}\left[\frac{\operatorname{sgn} z}{\sqrt{1+\frac{1}{2}\left(\frac{a}{z}\right)^{2}}}-1\right] . \\
\varphi & \equiv \ell_{+}=-\frac{G \lambda}{c^{4}}\left[\frac{\operatorname{sgn} z}{\sqrt{1+\frac{1}{2}\left(\frac{a}{z}\right)^{2}}}-1\right] \\
& =\frac{G \lambda}{c^{4}}\left[1-\frac{\operatorname{sgn} z}{\sqrt{1+\left(\frac{1}{2} \frac{a}{z}\right)^{2}}}\right] .
\end{aligned}
$$

Note that this result is positive-definite, hence regardless of the orientation of the light ray, the polarization changes in the same direction as the circulation of the ring laser. Allowing the $z$ to decrease without bound yields the now familiar result

$$
\Delta \phi=\frac{2 G \lambda}{c^{4}}
$$

Thus, when the beam traverses in the opposite direction, the shift in polarization angle is the same as above, so if the original incident beam was reflected, we see an amplification of the shift. As a result, allowing an incident beam to be reflected at both ends will produce an amplification of the gravitational Farday effect which we hope to be able to measure this amplified change of polarization angle by making use of confocal Fabrey-Perot (FP) laser technology. An acurate measurement of 
this polarization shift would be evidence of the gravitational frame dragging effect due to the ring laser.

\section{A CONCEPTUAL LABORATORY EXPERIMENT}

In this section we describe the feasibility of conducting a possible laboratory experiment to measure the gravitational Faraday rotation of a polarized laser beam that passes through a long light tunnel made out by stacking a very large number of ring lasers. Actual ring lasers are expensive, difficult to make and difficult to manipulate their size. So, we have conceived the idea of simulating a functional ring laser by arranging four independent commercial lasers placed on the same plane arranged in such a way that an effective ring laser is formed at the center where the four beams cross through each other in pairs (Figure 2). Symmetrically focused four lasers from four corners can simulate the effect of a high intensity ring laser beam with a very small cross-section to generate space twisting effect at the center. Use of high power diode lasers allows one to make the system very compact. The linear power density of the ring laser arm needs to be as high as possible to increase the Faraday rotation of the beam passing through the center of the ring laser plane orthogonally. Since making a very high power laser is nonlinearly expensive, we have chosen to enhance the effect by creating a tunnel of $N$-ring lasers through which the polarized beam travels. Conceptually the ring laser tunnel can be understood from Figure 3 which is simply a stack of $N$ independent ring lasers as depicted in Figure 2. The cumulative effect of gravitational space twisting can be multiplied $N$ times by stacking $N$ number of 4-laser diode rings side by side. 
Costwise, semiconductor diode lasers are potentially very good candidates for the construction of our proposed laboratory experimental concept. We have carried out a preliminary experiment ${ }^{(5)}$ to demonstrate that a compactly soldered stack of ten diode lasers can be operated to emit very high peak power, which can be even suitable for laser material processing. Four such stacks, when properly aranged would create a small tunnel of ten ring lasers. It is now easy to visualize how one can create a much longer light tunnel of ring lasers. Actual calculations are carried out in the cited reference ${ }^{(5)}$.

Finally, (Figure 4) gives the experimental setup for the actual space twisting tunnel placed at the center of the ring laser. One possible conceptual arrangement for the measurement of the gravitational Faraday rotation consists of utilizing a long and very high finesse confocal Fabry-Perot interferometer (FP) depicted by the mirrors $\mathrm{M}_{1}$ and $\mathrm{M}_{2}$. The polarized beam to undergo Faraday rotation passes through $M$ segments of ring laser tunnels ( $\mathrm{T}_{M}$ 's) placed inside the FP. A high finesse FP enhances the Faraday rotation even further due to multiple reflection of the beam. The necessary mathematical relation for such multiple reflections will be presented elsewhere. The lenses $\mathrm{L}_{M}$ 's are designed to keep the polarized beam "mode matched" within the FP while keeping the beam smaller than the ring laser tunnels. The advantage of this intracavity mode matching lenses $\mathrm{T}_{M}$ 's is that one can insert a very large number of $M$ ring laser tunnels $\mathrm{T}_{M}$ 's by folding the series into a three dimensional spiral ring with the help of flat mirrors (not shown in the Figure 5) to further enhance the Faraday rotation. 


\section{CONCLUSION}

This paper has shown that the gravitational field produced by the circulating light beam of a ring laser results in the rotation of the plane of polarization of an electro-

magnetic wave. A laboratory arrangement has been suggested that should allow the experimental verification of the predicted ring laser-gravitational Faraday effect. In this way, a novel optical-gravitational means of demonstrating general relativistic frame dragging will have been achieved. 


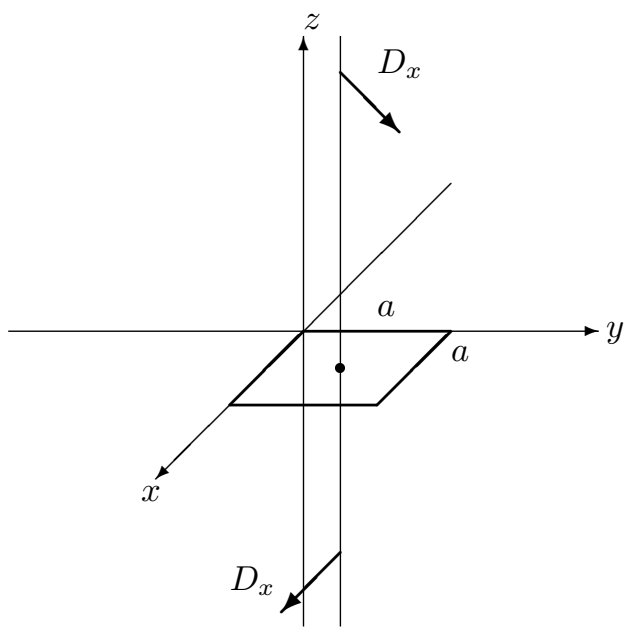

Figure 1 


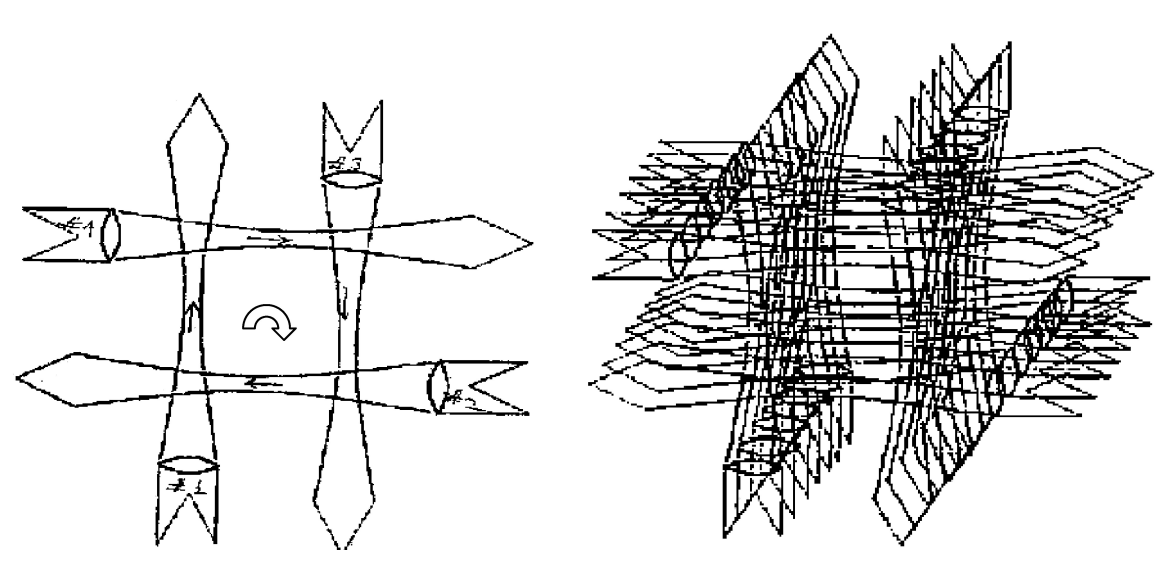

Figure 2 


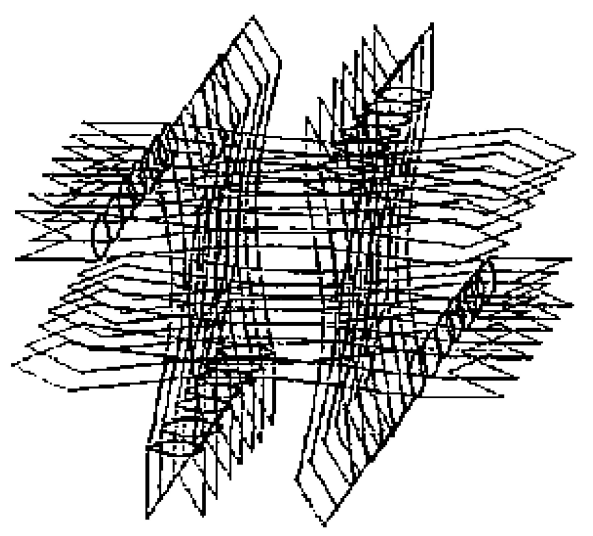

Figure 3 


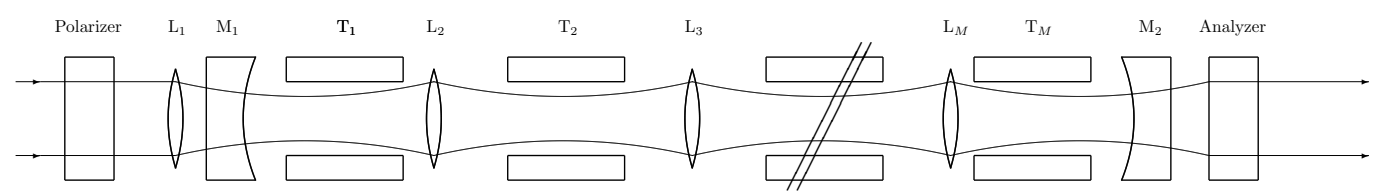

Figure 4 
Figure Headings

(1) Setup

(2) Single Ring Configuration

(3) Ring Stack Configuration

(4) Space Twisting Tunnel

\section{REFERENCES}

[1] Ciufolini, I. and Wheeler, J.A. Gravitation and Inertia, Chapter 5. Princeton University Press. 1995.

[2] Mallett, Ronald L. Weak Gravitational Field of the Electromagnetic Radiation in a Ring Laser. Physics Letters A 269 (2000) 214-217.

[3] Balazs, N.L. Effect of a Gravitational Field, Due To a Rotating Body, on the Plane of Polarization of an Electromagnetic Wave. Physical Review. Volume 11, Number 1. 1958.

[4] Landau, L.D. and E.M. Lifshitz. The Classical Theory of Fields. Fourth Revised English Edition. Butterworth-Heinemann. 2000.

[5] Stelmakh, N., Lopez, J., Smolski, O. and Roychoudhuri, C., June 2000, 100W 50ps gain switched pulses from vertical- stack laser diode, Electronics Letters, Vol. 36, No.12, pp 102224 . 